(C) Masson, Paris, 1982 .

\title{
TROIS CAS D'ANOMALIES MORPHOLOGIQUES DONT UN GYNANDROMORPHISME CHEZ SERGENTOMYIA MINUTA ET S. DENTATA (DIPTERA, PHLEBOTOMIDAE) DE GRËE
}

\author{
N. LEGER*, B. PESSON**, G. MADULO-LEBLOND*** et J. COLLOMB****
}

RÉSUMÉ. Les auteurs rapportent 3 cas d'anomalies morphologiques chez des Phlébotomes récoltés dans l'île de Corfou et discutent l'emploi du terme " gynandromorphisme ».

\section{Three cases of abnormality in sandflies from Corfou (Greece)}

SUMMARY. The authors describe 3 eases of abnormality in sandflies collected in Corfou island and argue about the use of the word "gynandromorphism".

Bien que peu fréquentes des anomalies morphologiques sont régulièrement signalées chez les Phlébotomes. En 1971, Abonnenc et coll., en font l'inventaire et donnent une bibliographie exhaustive.

Le plus souvent il s'agit d'épines surnuméraires au niveau du style de l'appareil copulateur mâle ( 36 cas). Les anomalies du cibarium sont plus rarement rapportées. Quant au gynandromorphisme il apparaît comme exceptionnel : en 1977, Addadi et Dedet en citent 5 cas. Il s'agit toujours d'un appareil génital mâle anormal associé ou non avec des éléments femelles, c'est-à-dire d'intersexualité. Le gynandromorphisme extra génital n'est pas toujours individualisé au sein des anomalies morphologiques. Ainsi Parrot et Habibi (1946) ne parlent pas de gynandromorphisme à propos d'une femelle de $P$. minutus présentant un cibarium faiblement armé, caractère généralement associé au sexe mâle.

Lors d'une prospection entomologique effectuée en 1979-1980 dans l'île de Corfou (Grèce) et au cours de laquelle nous avons récolté 21914 phlébotomes, nous avons observé 3 exemplaires anormaux :

* U.E.R. de Pharmacie de Reims, F51096 Reims.

** U.E.R. de Pharmacie de Strasbourg, F67048 Strasbourg.

*** U.E.R. de Pharmacie de Paris XI, F92290 Chatenay Malabry.

**** U.E.R. de Pharmacie de Nancy, F54000 Nancy.

Accepté le I2 juin $198 \mathrm{r}$. 


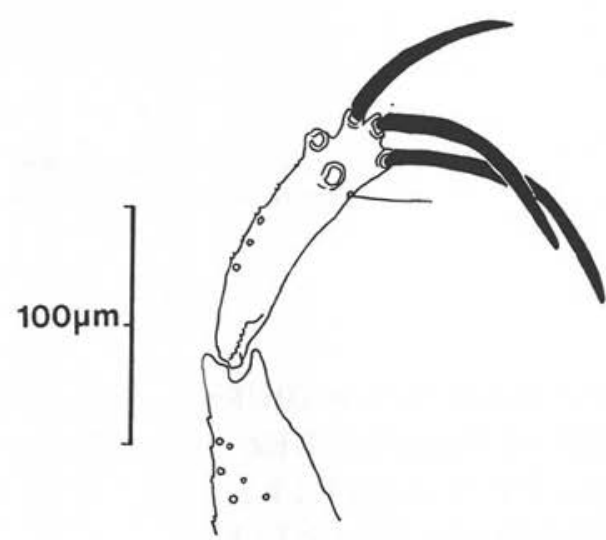

FIG. I. - Sergentomyia minuta (Rondani, r843) mâle : style à 5 épines terminales.

FIG. 2. - Sergentomyia dentata (Sinton, 1933) femelle : cibarium inerme. $\rightarrow$
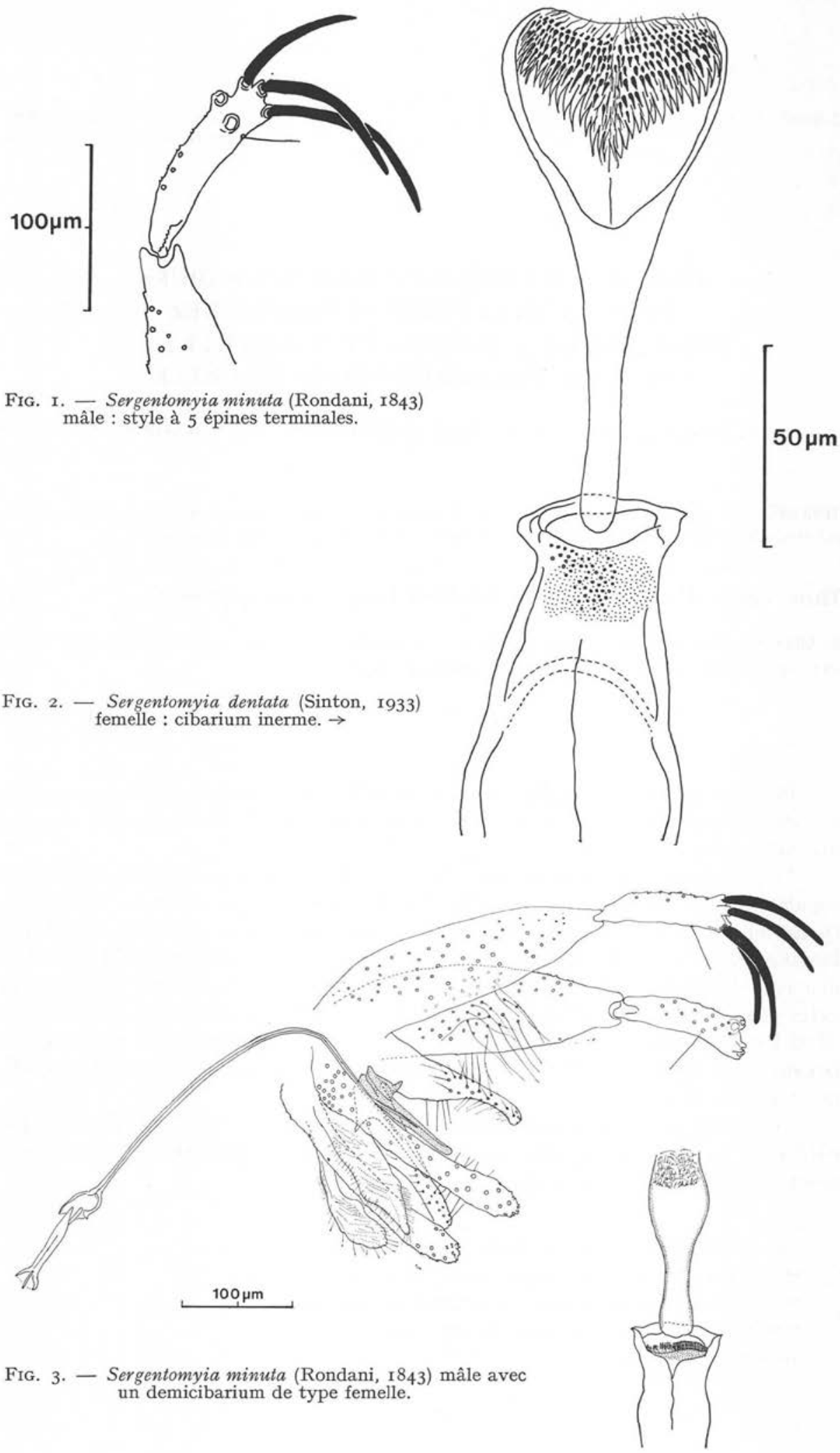
1 - un Sergentomyia minuta mâle dont l'un des styles portait 5 épines terminales au lieu de 4 ( fig. 1).

2 - un Sergentomyia dentata femelle possédant un cibarium totalement inerme (fig. 2).

3 - un Sergentomyia minuta mâle avec un demi cibarium de type femelle alors que l'autre moitié était normale (fig. 3).

Le terme de gynandromorphisme nous semble devoir s'appliquer à l'anomalie présentée par l'exemplaire no 3 bien qu'il ne s'agisse pas d'intersexualité puisque l'appareil génital mâle était strictement normal.

\section{BIBLIOGRAPHIE}

Abonnenc E., Poinsot S., Rioux J. A. : Tératologie des Phlébotomes (Diptera: Psychodidae). Révision et nouvelles observations. Cah. ORSTOM, sér. Ent. méd. et Parasitol., 1971, 9, 307-316.

ADDADI K., DEDET J. P. : Un nouveau cas de gynandromorphisme chez Sergentomyia minuta parroti (Adler et Theodor, 1927) (Diptera, Psychodidae), Arch. Inst. Pasteur Algér., 1977, 52, $135-138$.

PARROT L., HABIBI A. : Notes sur les Phlébotomes LIV. Formes anormales de Phlebotomus minutus var. parroti Adler et Theodor, 1927. Arch. Inst. Pasteur Algér., 1946, 24 , 157-1 59.

Cette étude bénéficie d'un appui financier du Programme spécial PNUD/Banque mondiale/ OMS pour la recherche et la Formation concernant les maladies tropicales. 